\title{
Everolimus sensitizes Ras-transformed cells to radiation in vitro through the autophagy pathway
}

\author{
YU-CHIEH SU ${ }^{1,6^{*}}$, CHIH-CHIA YU ${ }^{1,2^{*}}$, FEI-TING HSU ${ }^{3}$, SHU-LING FU ${ }^{4}$, JENG-JONG HWANG $^{3}$, \\ LING-CHIEN HUNG ${ }^{4}$, MOON-SING LEE ${ }^{5,6}$, WEN-YEN CHIOU ${ }^{5,6}$, HON-YI LIN ${ }^{5,6}$ and SHIH-KAI HUNG ${ }^{5,6}$ \\ ${ }^{1}$ Department of Hematological Oncology, Buddhist Dalin Tzu Chi Hospital, Chiayi 62247; \\ ${ }^{2}$ Department of Life Science and Institute of Molecular Biology, National Chung-Cheng University, Chiayi 62102; \\ ${ }^{3}$ Department of Biomedical Imaging and Radiological Sciences, National Yang-Ming University, Taipei 11221; \\ ${ }^{4}$ Institute of Traditional Medicine, National Yang-Ming University, Taipei 11221; ${ }^{5}$ Department of Radiation Oncology, \\ Buddhist Dalin Tzu Chi Hospital, Chiayi 62247; ${ }^{6}$ School of Medicine, Tzu Chi University, Hualian 97061, Taiwan, R.O.C.
}

Received May 15, 2014; Accepted September 4, 2014

DOI: $10.3892 / \mathrm{ijmm} .2014 .1927$

\begin{abstract}
Modern radiation therapy strives to minimize injury to organs while increasing the anticancer effects. The present study aimed to investigate the radiosensitizing effects of everolimus and to examine the molecular mechanisms responsible for everolimus-mediated radiosensitization. Radiation in combination with everolimus (30 nM) sensitized Ras-transformed cells to radiation in vitro. Radiation induced apoptotic markers (sub-G1 cell accumulation, membrane inversion and DNA fragmentation) and treatment with everolimus did not promote radiation-induced apoptosis. However, LC3-II expression increased following combination treatment with everolimus and radiation, and the radiosensitizing effects of everolimus were reversed following transfection with small interfering RNA (siRNA) targeting Beclin 1. In addition, the protein levels of activated S6 kinase 1 (S6K1) were significantly reduced following treatment with everolimus, and the phosphorylation of factor 4E binding protein 1 (4EBP1) was suppressed following combination treatment. Taken together, our data demonstrate that everolimus sensitizes Ras-transformed cells to radiation in vitro. Everolimus-mediated radiosensitization is associated with the autophagy pathway. Thus, everolimus is a novel radiosensitizing agent with potential for use in cancer radiotherapy.
\end{abstract}

\section{Introduction}

Malignant tumors are characterized by uncontrolled growth, invasion and metastasis. The elevated expression and activa-

Correspondence to: Dr Shih-Kai Hung, Department of Radiation Oncology, Buddhist Dalin Tzu Chi General Hospital, 2 Ming Sheng Road, Dalin, Chiayi 62247, Taiwan, R.O.C.

E-mail: oncology158@yahoo.com.tw

*Contributed equally

Key words: autophagy, everolimus, Ras, radiosensitization tion of oncogenes are strongly associated with the initiation and progression of cancer. Ras, one of the well-characterized proto-oncogenes, controls multiple intracellular signaling networks (1). Aberrant Ras activation is highly associated with cancer initiation, as well as progression, and its presence has been shown in 20-30\% of all human tumors, particularly in colon and pancreatic cancer $(2,3)$.

Radiation therapy is an important therapeutic strategy in cancer treatment. Minimizing injury to the surrounding normal tissue and organs, while increasing the anticancer effects remains an unresolved issue in the field of radiation therapy. Ras has been studied and linked to radioresistance. A number of studies have reported that the inhibition of Ras activation by anti-Ras antibody or Ras inhibitors can enhance radiation-induced cell death in human tumor cell lines $(4,5)$. The involvement of the phosphoinositide 3-kinase (PI3K)/ protein kinase $\mathrm{B}(\mathrm{Akt})$ signaling pathway in Ras-induced radioresistance suggests that the inhibition of PI3K/Akt activity can greatly enhance the radiosensitivity of Ras-transformed cells (6). Everolimus is an inhibitor of mammalian target of rapamycin (mTOR) and a derivative of rapamycin with immunosuppressiveand anticancer properties $(7,8)$. The anticancer effects of everolimus are associated with the inhibition of the $\mathrm{PI} 3 \mathrm{~K} / \mathrm{AKT} / \mathrm{mTOR}$ pathway $(8,9)$. Thus, everolimus may act as a radiosensitizer in Ras-transformed cells. The present study aimed to investigate the radiosensitizing effects of everolimus and to elucidate the molecular mechanisms underlying everolimus-mediated radiosensitization.

\section{Materials and methods}

Chemicals and antibodies. Everolimus was obtained from Novartis (Basel, Switzerland) and dissolved in dimethyl sulfoxide (DMSO) as a concentrated stock solution. DMSO was used as the vehicle solution. As an internal control, medium only was used. DMSO and anti-actin antibody were from Sigma-Aldrich (St. Louis, MO, USA). Antibodies against Ras, phospho-eukaryotic initiation factor 4E-binding protein 1 (p-4EBP1), 4EBP1, phospho-ribosomal protein S6 
kinase 1 (p-S6K1), S6K1 and phospho-Akt (p-Akt; Ser-473) were from Upstate Biotechnology (Lake Placid, NY, USA). Antibodies against phospho-mTOR (p-mTOR), mTOR, Akt and eukaryotic translation initiation factor $4 \mathrm{E}$ (eIF4E) were from BD Biosciences (San Diego, CA, USA). Beclin 1 small interfering RNA (siRNA) was from Thermo Fisher Scientific (Carlsbad, CA, USA).

Cell culture. RK3E/tv-a cells were obtained from the laboratory of Shu-Ling Fu, who established a non-transformed rat kidney epithelial RK3E cell line that constitutively expresses tv-a (receptor for subgroup A avian leukosis virus, ALV) for the delivery of foreign genes via avian retroviral infection. The $\mathrm{RK} 3 \mathrm{E} / \mathrm{tv}$-a cells were infected with retrovirus carrying the oncogene, $\mathrm{H}$-ras, or the control gene puromycin $\mathrm{N}$-acetyltransferase (puro) as previously described (10). Ras-expressing cells, but not puro-expressing cells, underwent malignant transformation in vitro and tumorigenesis in vivo. These cells were maintained as previously described (10).

MTT assays. 3-(4,5-Dimethyl-2-thiazolyl)-2,5-diphenyl-2H tetrazolium bromide (MTT) assays were carried out to measure the cytotoxic and radiosensitizing effects of everolimus. Ras-transformed cells $\left(5 \times 10^{3}\right)$ were seeded into 96-well plates, incubated overnight, treated with everolimus and/or radiation and cultured for 4 days. The medium was removed and the cells were incubated with MTT solution $(0.5 \mathrm{mg} / \mathrm{ml}, 4 \mathrm{~h}$, overnight, $5 \% \mathrm{CO}_{2}$ incubator, $37^{\circ} \mathrm{C}$ ) and lysed with solubilization solution [12.5\% sodium dodecyl sulfate (SDS), $45 \%$ dimethylformamide (DMF)]. The absorbance of converted dye was measured at $570 \mathrm{~nm}$ using a microplate reader. (Multiskan RC, Labsystems, Stockholm, Sweden). The absorbance of the untreated cells measured at $570 \mathrm{~nm}$ was defined as $100 \%$. The relative viability was calculated by dividing the A570 $\mathrm{nm}$ of the treated cells by that of the untreated cells.

siRNA-mediated knockdown of Beclin 1. The Ras-transformed cells $\left(4 \times 10^{5}\right)$ were seeded into a 6 -cm dish, cultured overnight, and transfected at $40 \%$ confluency with the indicated specific constructs (Beclin 1 siRNA, or non-targeted control siRNA) for $24 \mathrm{~h}$ according to the manufacturer's instructions (Thermo Scientific Dharmacon Inc., Lafayette, CO, USA). These sequences were 5'-GGACAGUUUGGCACAAUCA-3' (951-969). For each transfection, 2 solutions (one of $3 \mu 1$ of siRNA diluted in $100 \mu \mathrm{l}$ transfection medium and another of $3 \mu \mathrm{l}$ of transfection reagents diluted in $100 \mu \mathrm{l}$ transfection medium) were combined at room temperature for $20 \mathrm{~min}$ and added to each well, and the wells were incubated for $24 \mathrm{~h}$. The transfection efficiency was determined at $24 \mathrm{~h}$ by western blot analysis. MTT assays were used to measure the effects of everolimus-mediated radiosensitization.

Annexin Vstaining. An Annexin V-FITC kit was used according to the manufacturer's instructions (R\&D Systems Inc., Minneapolis, MN, USA). In brief, the cells $\left(10^{5}\right)$ were grown on 6-well plates, treated with the vehicle (DMSO) or everolimus $(10 \mathrm{nM})$ with or without $6 \mathrm{~Gy}$ radiation, cultured for $96 \mathrm{~h}$ and stained with Annexin V-FITC and propidium iodide (PI) $(5 \mu \mathrm{g} / \mathrm{ml})$. The stained cells were analyzed using a flow cytometer (FACScalibur; Becton-Dickinson, San Jose, CA, USA).
Terminal deoxynucleotidyl transferase-mediated dUTP nickend labeling (TUNEL) assay. A (TUNEL) assay was performed to measure cell death according to the manufacturer's instructions (Roche, Mannheim, Germany). The cells $\left(10^{5}\right)$ were seeded into 6-well plates, incubated overnight and subsequently treated with the vehicle, everolimus and/or radiation under various conditions. Following treatment, the floating and adherent cells were collected and fixed with $4 \%$ paraformaldehyde in PBS, permeablized with $0.1 \%$ Triton X-100, stained with TUNEL reaction mixture for $60 \mathrm{~min}$ and analyzed by flow cytometry using a FACScan flow cytometer (Becton-Dickinson).

Cell cycle analysis. Cells $\left(10^{5}\right)$ were grown on 6-well plates, treated under various conditions, incubated for $96 \mathrm{~h}$ at $37^{\circ} \mathrm{C}$, then trypsinized, washed, fixed in $80 \%$ cold ethanol for $30 \mathrm{~min}$, stained with $50 \mu \mathrm{g} / \mathrm{ml}$ propium iodide in PBS containing $50 \mu \mathrm{g} / \mathrm{ml}$ of RNase A for $30 \mathrm{~min}$, and immediately analyzed by flow cytometry using a FACScan flow cytometer.

Western blot analysis. The cells $\left(10^{6}\right)$ were seeded into $100-\mathrm{mm}$ plates, incubated overnight, treated with the vehicle, everolimus or radiation under various conditions, and collected by scraping and centrifugation at 3,000 rpm for $5 \mathrm{~min}$. The cell pellets were then lysed in radioimmune precipitation assay buffer (RIPA) in the presence of general protease inhibitors. Total protein (50 $\mu \mathrm{g}$ of lysate proteins) was analyzed by $10 \%$ sodium dodecyl sulfate polyacrylamide-gel electrophoresis (SDS-PAGE) and transferred onto PVDF membranes. After blocking with $5 \%$ non-fat milk/TBS-Tween, the membranes were incubated with primary antibodies, including anti-pAkt, anti-Akt, anti-p-mTOR, anti-mTOR, anti-phospho-S6K (p-S6K), anti-S6K, anti-p-4EBP1, anti-4EBP1, anti-eIF4E and anti-LC3 antibodies overnight at $4^{\circ} \mathrm{C}$, reacted with horseradish peroxidase (HRP)-conjugated secondary antibodies for $1 \mathrm{~h}$, treated with Immobilon ${ }^{\mathrm{TM}}$ Western Chemiluminescent HRP Substrate (ECL) and exposed to X-ray film to detect the signals.

Statistical analysis. Data are expressed as the means \pm SD of 3independent experiments. Statistical analyses were performed using the unpaired two-tailed Student's t-test to compare 2 groups of data. A value of $\mathrm{P}<0.05$ was considered to indicate a statistically signficant difference.

\section{Results}

Everolimus-mediated radiosensitization of Ras-transformed cells in vitro. We first evaluated the cytotoxicity of everolimus to Ras-transformed cells by MTT assay. The cytotoxic effects were found to increase with the higher everolimus concentrations. The survival fraction of the cells treated with everolimus was $(10,30$ and $50 \mathrm{nM})$ was $0.91 \pm 0.03,0.84 \pm 0.04$ and $0.8 \pm 0.02$, respectively. Thus, everolimus ( $30 \mathrm{nM} ; 96 \mathrm{~h}$ of incubation) was used in the subsequent radiosensitivity experiments. As shown in Fig. 1, treatment with everolimus $(30 \mathrm{nM})$ sensitized the Ras-transformed cells to radiation (6 Gy). The survival fractions of the irradiated cells in the medium, vehicle and everolimus solution were $0.45 \pm 0.04,0.42 \pm 0.04$ and $0.12 \pm 0.04$, respectively.

Everolimus does not promote radiation-induced apoptosis. We wished to determine whether everolimus, with or 
Table I. Cell cycle distribution under various treatment conditions.

\begin{tabular}{lrrrrrr}
\hline Phase & \multicolumn{1}{c}{ M0 } & M6 & \multicolumn{1}{c}{ D0 } & \multicolumn{1}{c}{ D6 } & R0 & R6 \\
\hline Sub- $\mathrm{G}_{1}$ & $2.9 \pm 0.8$ & $7.1 \pm 2.0$ & $3.1 \pm 0.3$ & $7.8 \pm 4.1$ & $2.2 \pm 1.4$ & $5.8 \pm 2.9$ \\
$\mathrm{G}_{0} / \mathrm{G}_{1}$ & $64.9 \pm 6.4$ & $47.5 \pm 6.1$ & $66.0 \pm 4.8$ & $48.1 \pm 6.0$ & $62.0 \pm 3.7$ & $49.4 \pm 6.3$ \\
$\mathrm{~S}$ & $14.3 \pm 2.3$ & $15.2 \pm 1.8$ & $13.3 \pm 0.9$ & $14.5 \pm 1.2$ & $13.9 \pm 0.7$ & $13.9 \pm 1.4$ \\
$\mathrm{G}_{2} / \mathrm{M}$ & $18.1 \pm 4.6$ & $30.5 \pm 3.1$ & $18.1 \pm 4.6$ & $30.0 \pm 2.6$ & $22.3 \pm 2.9$ & $31.3 \pm 2.9$ \\
\hline
\end{tabular}

M0: medium, 0 Gy; M6: medium, 6 Gy; D0: 0.1\% DMSO, 0 Gy; D6: 0.1\% DMSO, 6 Gy; R0: everolimus (30 nM), 0 Gy; R6: everolimus $(30 \mathrm{nM}), 6 \mathrm{~Gy}$.

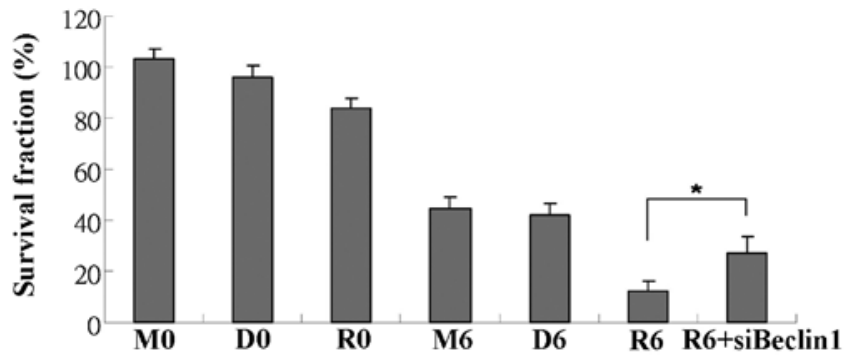

Figure 1. Everolimus sensitizes Ras-transformed cells to radiation in vitro. MTT assay was used to determine the radiosensitizing effects of everolimus. The cell survival fractions were calculated, and the calculated cell survival fractions following 6 Gy of radiation and treatment with medium only, vehicle (DMSO) or everolimus solution (stock diluted appropriately in medium) were $0.45 \pm 0.04,0.42 \pm 0.04$ and $0.12 \pm 0.04$, respectively. In addition, the radiosensitizating effects of everolimus were reversed following transfection with Beclin 1 siRNA. "P<0.05. M0: medium, 0 Gy; M6: medium, 6 Gy; D0: 0.1\% DMSO, 0 Gy; D6: 0.1\% DMSO, 6 Gy; R0: everolimus (30 nM), 0 Gy; R6: everolimus (30 nM), 6 Gy radiation.

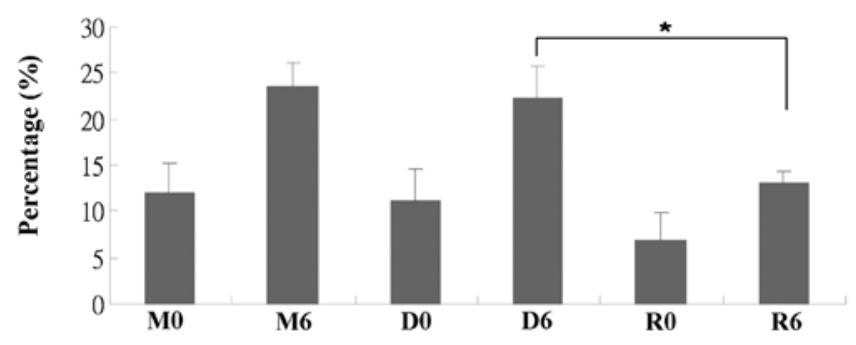

Figure 2. Everolimus does not promote radiation-induced apoptosis. The Ras-transformed cells were treated with everolimus $(10 \mathrm{nM})$ with or without radiation (6 Gy), incubated for $96 \mathrm{~h}$, and stained with Annexin V-FITC and propidium iodide. Radiation increased the percentage of Annexin V-stained cells, and everolimus in combination with radiation decreased the percentage of apoptotic cells. "P<0.0. M0: medium, 0 Gy; M6: medium, 6 Gy; D0: 0.1\% DMSO, 0 Gy; D6: 0.1\% DMSO, 6 Gy; R0: everolimus (30 nM), 0 Gy; R6: everolimus (30 nM), 6 Gy.

without radiation, induces apoptosis. Thus, we performed Annexin V staining and TUNEL assay. The percentage of Annexin V-stained cells was increased following radiation, but not following treatment with everolimus (Fig. 2). Consistent with the above observations, DNA fragmentation (measured by TUNEL assay) was mainly caused by radiation (Fig. 3). By contrast, combination treatment with everolimus and radiation decreased the percentage of dead/apoptotic cells. Taken

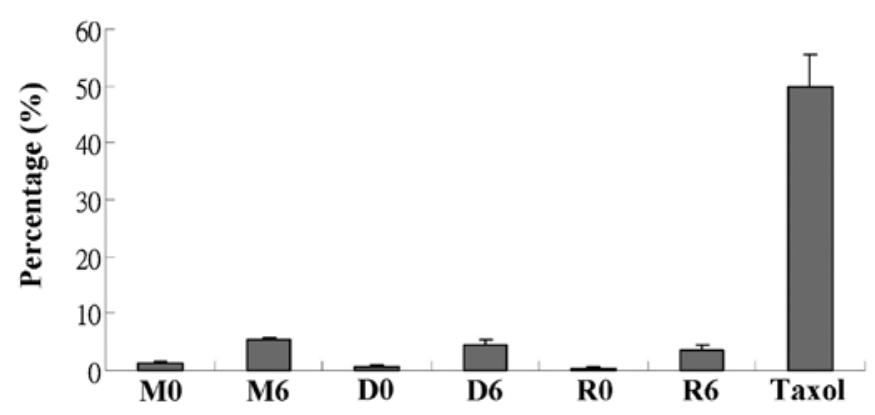

Figure 3. Everolimus does not promote radiation-induced apoptosis. The Ras-transformed cells were treated with everolimus $(10 \mathrm{nM})$ with or without radiation ( $6 \mathrm{~Gy})$, incubated for $96 \mathrm{~h}$ and stained with TUNEL. DNA fragmentation was caused by radiation. ${ }^{*} \mathrm{P}<0.05$. M0: medium, 0 Gy; M6: medium, 6 Gy; D0: 0.1\% DMSO, 0 Gy; D6: 0.1\% DMSO, 6 Gy; R0: everolimus (30 nM), 0 Gy; R6: everolimus (30 nM), 6 Gy.

together, these data indicate that apoptosis is mainly induced by radiation and not is promoted by everolimus. The decreased percentage of apoptotic cell death suggests that everolimusmediated radiosensitization leading to cell death may occur through a mechanism other than apoptosis.

Radiation affects cell cycle distribution. We wished to determine whether everolimus, with or without radiation, affects cell cycle distribution. Thus, we used PI staining followed by flow cytometry using a FACScan flow cytometer. As shown in Table I, radiation significantly increased the size of the sub- $\mathrm{G}_{1}$ and $G_{2} / M$ fractions $(P<0.05)$, and radiation in combination with everolimus (compared to radiation alone) only slightly decreased the size of the sub- $\mathrm{G}_{1}$ fraction.

LC3-II expression increases following treatment with everolimus in combination with radiation and the radiosensitizing effects of everolimus are reversed by transfection with Beclin 1 siRNA. We wished to determine whether everolimus, with or without radiation, induces autophagy. Thus, we performed western blot analysis. Fig. 4 shows the protein expression of LC3-II under various conditions. Everolimus in combination with radiation significantly increased LC3-II expression. We then wished to determine whether the everolimus-mediated radiosensitizing effects were dependent on autophagy. Thus, we transfected the cells with Beclin 1 siRNA. As shown in Fig. 1, the radiosensitization effects of everolimus were partially reversed following transfection with Beclin 1 siRNA. 


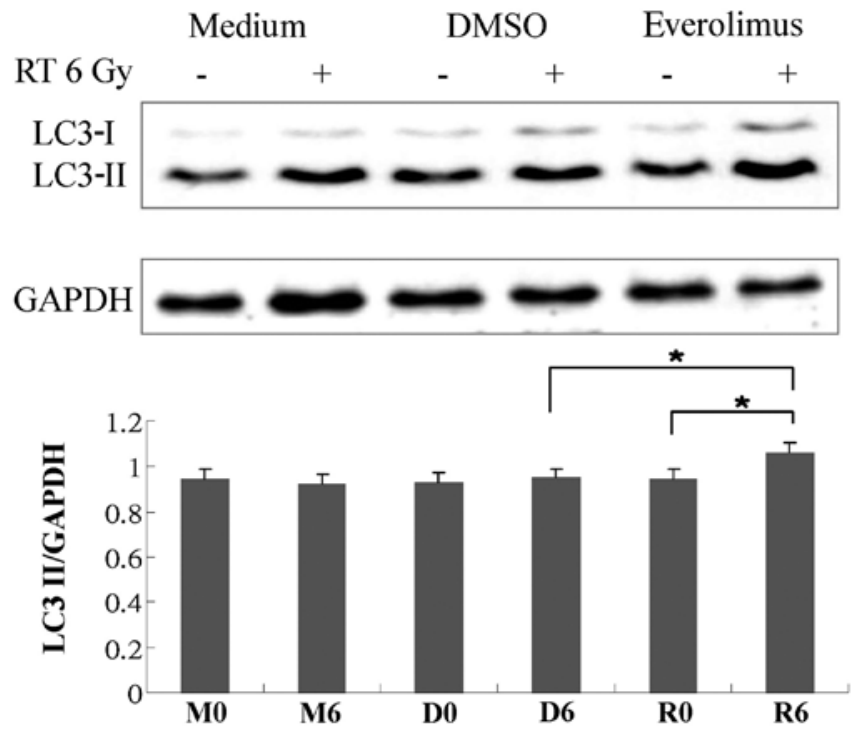

Figure 4. LC3-II expression is increased following combination treatment with everolimus and radiation. (Upper panel) representative western blots showing the protein expression of LC3-I and LC3-II under various treatment conditions. GAPDH was used as the loading control. (Bottom panel) Quantitative analysis of results from western blot analysis. For each treatment, the protein levels of LC3-II were normalized to those of GAPDH. "P<0.05. M0: medium, 0 Gy; M6: medium, 6 Gy; D0: 0.1\% DMSO, 0 Gy; D6: 0.1\% DMSO, 6 Gy; R0: everolimus ( $30 \mathrm{nM}), 0$ Gy; R6: everolimus (30 nM), 6 Gy.
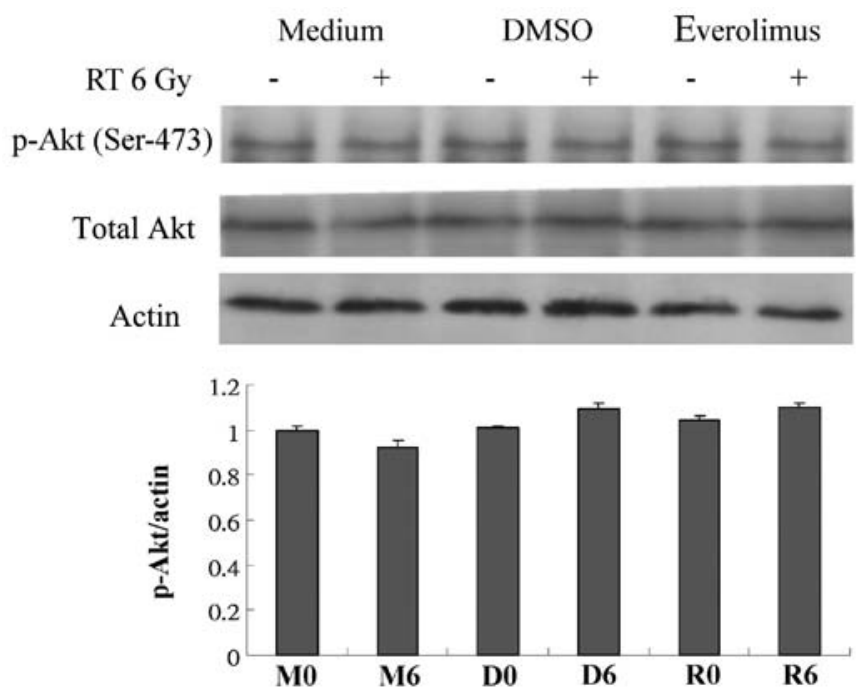

Figure 5. Akt protein expression is not altered by everolimus or radiation. (Upper panel) Representative western blots show the protein expression of p-Akt (Ser473) and Akt under various treatment conditions. Actin was used as the loading control. (Bottom panel) Quantitative analysis of the results from western blot analysis. For each treatment, the protein levels of p-Akt were normalized to those of actin. M0: medium, 0 Gy; M6: medium, $6 \mathrm{~Gy}$; D0: 0.1\% DMSO, 0 Gy; D6: 0.1\% DMSO, 6 Gy; R0: everolimus (30 nM), 0 Gy; R6: everolimus (30 nM), 6 Gy.

Akt protein expression is not altered by everolimus or radiation. Since the PI3K/Akt pathway is a target of everolimus, the possible association of the radiosensitizing activity of everolimus with the PI3K/Akt signaling pathway was assessed. Western blot analysis revealed that the expression or activation of Akt protein was not significantly altered by everolimus or radiation (Fig. 5).

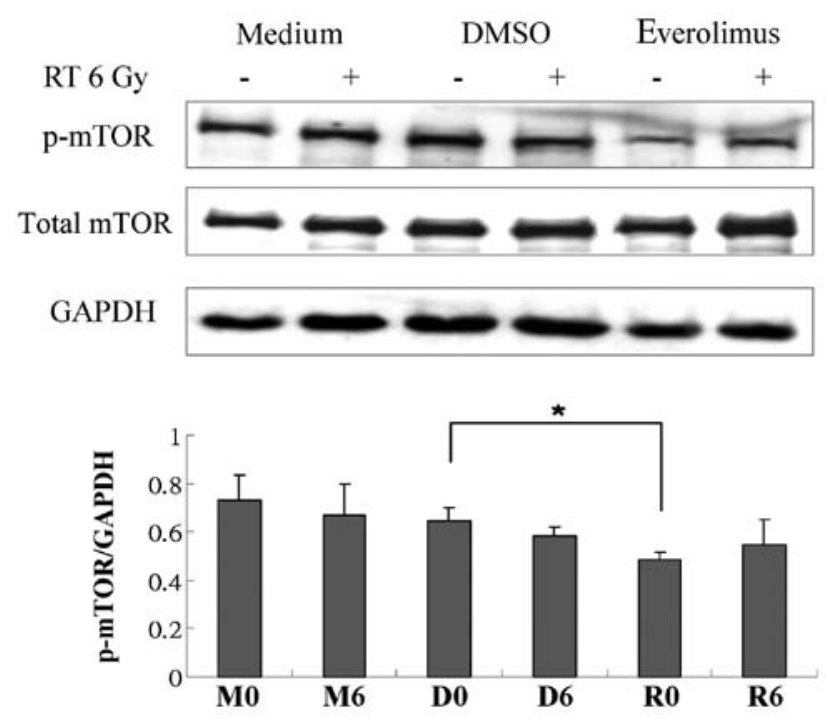

Figure 6. Treatment with everolimus suppresses the phosphorylation of mTOR. (Upper panel) Representative western blots show the protein expression of p-mTOR and mTOR under various treatment conditions. GAPDH was used as the loading control. (Bottom panel) Quantitative analysis of the results from western blot analysis. For each treatment, the protein levels of p-mTOR were normalized to those of GAPDH. ${ }^{*} \mathrm{P}<0.05$. M0: medium, 0 Gy; M6: medium, 6 Gy; D0: 0.1\% DMSO, 0 Gy; D6: 0.1\% DMSO, 6 Gy; R0: everolimus (30 nM), 0 Gy; R6: everolimus (30 nM), 6 Gy.

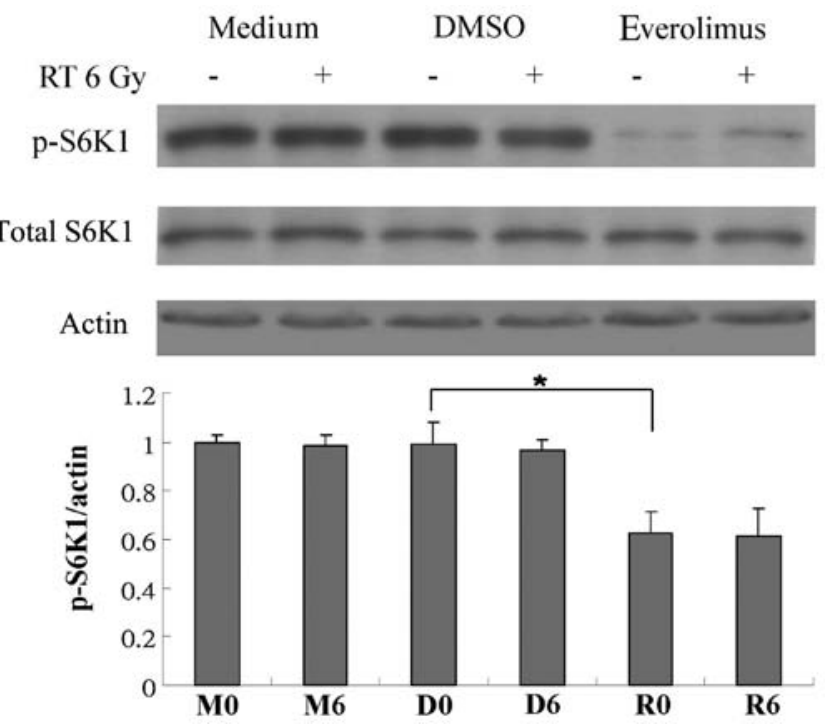

Figure 7. Treatment with everolimus suppresses S6K1 activation. (Upper panel) Representative western blots the protein expression levels of p-S6K1 and S6K1 under various treatment conditions. Actin was used as the loading control. (Bottom panel) Quantitative analysis of the results from western blot analysis. For each treatment, the protein levels of p-S6K1 were normalized to those of actin protein. ${ }^{*} \mathrm{P}<0.05$. M0: medium, 0 Gy; M6: medium, 6 Gy; D0: 0.1\% DMSO, 0 Gy; D6: 0.1\% DMSO, 6 Gy; R0: everolimus (30 nM), 0 Gy; R6: everolimus (30 nM), 6 Gy.

Phosphorylation of $4 E B P 1$ is suppressed following treatment with everolimus in combination with radiation. We wished to determine whether everolimus, with or without radiation, regulates the PI3K/AKT/mTOR pathway. Thus, we performed western blot analysis in order to determine the protein expression of p-mTOR, mTOR, p-S6K1, S6K1, p-4EBP1, 4BEP1 

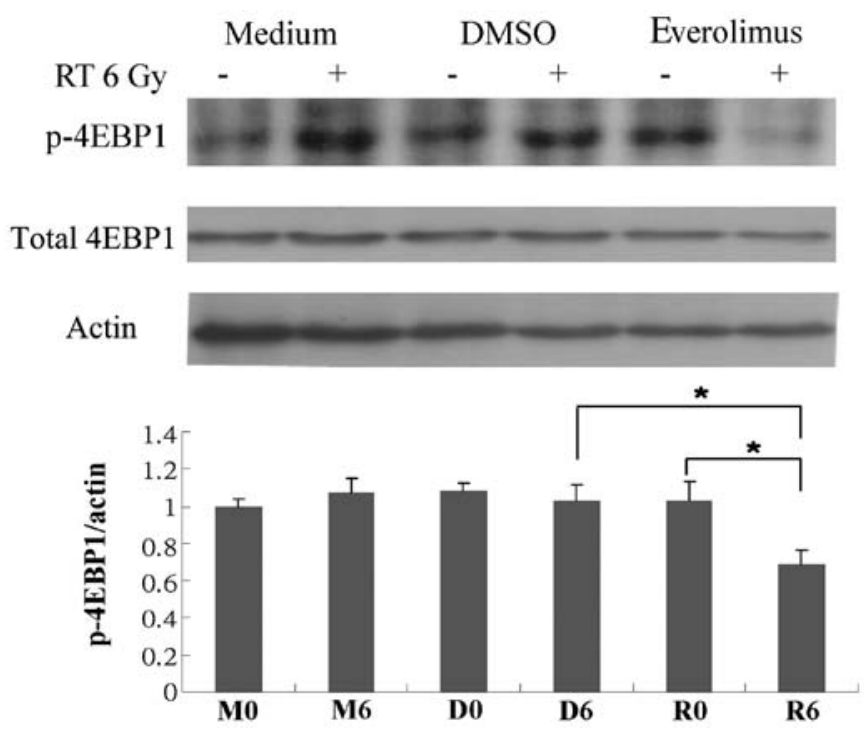

Figure 8. Phosphorylation of 4EBP1 is suppressed following combination treatment with everolimus and radiation. (Upper panel) Representative western blots show the protein expression of p-4EBP1 and 4EBP1 under various treatment conditions. Actin was used as the loading control. (Bottom panel) Quantitative analysis of the results from western blot analysis. For each treatment, the protein levels of $\mathrm{p}-4 \mathrm{EBP} 1$ were normalized to those of actin. ${ }^{*} \mathrm{P}<0.05$. M0: medium, 0 Gy; M6: medium, 6 Gy; D0: 0.1\% DMSO, 0 Gy; D6: 0.1\% DMSO, 6 Gy; R0: everolimus ( $30 \mathrm{nM}), 0$ Gy; R6: everolimus (30 nM), 6 Gy.

and eIF4E. There was no significant change observed in eIF4E expression (data not shown). However, treatment with everolimus alone attenuated the phosphorylation of mTOR and S6K1 (Figs. 6and 7), and everolimus in combination with radiation markedly suppressed the phosphorylation of 4EBP1 (Fig. 8).

\section{Discussion}

Activating Ras mutations are frequently observed in a number of human cancers and are strongly correlated with radiation resistance $(3,4,11)$. Colorectal cancer is one of the most frequently diagnosed cancers with aberrant Ras activation and a major cause of cancer-related mortality in Taiwan $(2,3,12)$. In the present study, we demonstrated the radiosensitizing activity of everolimus in Ras-transformed cells, suggesting a potential new application of this compound in cancer therapy.

Different types of cell death with possible associations to everolimus-mediated radiosensitization were examined. Our results revealed that radiation-induced apoptotic changes (sub- $\mathrm{G}_{1}$ cell accumulation, membrane inversion and DNA fragmentation) were not enhanced by treatment with everolimus (Table I; Figs. 2 and 3). Notably, the percentage of apoptotic cells decreased following treatment with everolimus combined with radiation relative to the percentage following radiation alone, suggesting that other non-apoptotic pathways may be involved in the everolimus-mediated radiosensitization. Indeed, increasing evidence indicates that non-apoptotic mechanisms, such as autophagy, are crucial for treatmentinduced tumor-cell death $(13,14)$. Whether the cell death caused by everolimus-mediated radiosensitization is induced by autophagy was examined by assessing the protein expression of LC3. Western blot analysis revealed that treatment with everolimus plus radiation markedly increased LC3-II expression (Fig. 4). In addition, siRNA targeting Beclin 1 was used to further determine whether the everolimus-mediated radiosensitizing effects are dependent on autophagy. As shown in Fig. 1, the radiosensitizing effects of everolimus were reversed following transfection with Beclin 1 siRNA. These data indicate that the autophagy pathway is involved in the everolimus-mediated radiosensitizing effects.

Multiple signaling pathways, including the PI3K/AKT/ mTOR and Raf/MEK/ERK pathways, are associated with the pharmacological activity of everolimus $(15,16)$. The importance of the PI3K/AKT/mTOR pathway in tumor progression has been investigated (17). In addition, it has been shown that mTOR inhibitors target a number of cancers and regulate abnormal signaling (18). mTOR signaling is mediated through two multiprotein complexes, the mTOR complex 1 (mTORC1) and 2 (mTORC2). mTORC1 can activate 4EBP1 and S6K1 and lead to translation, increasing protein synthesis and growth (18). Several studies have demonstrated that mTOR inhibitors modulate autophagy. A previous study suggested that p-4EBP1 binds to and stabilizes mTORC1 in vitro (19). Another study demonstrated that the effects of 4EBP1 on autophagy were significant, as evidenced by the increased baseline levels of autophagy, the increased rapamycin-induced autophagy and the elimination of 4EBP1 in the presence of the autophagy inhibitor (20). Furthermore, S6K1 is sensitive to the modulation of autophagy and requires a much less stringent inhibition of MTORC1 to be targeted (21). In the present study, the combination of everolimus and radiation markedly suppressed the phosphorylation of 4EBP1 (Fig. 8). In addition, treatment with everolimus alone attenuated the phosphorylation of S6K1 (Fig. 7). These findings suggest that 4EBP1 and S6K1 interact with specific proteins involved in autophagy and may have as yet unrecognized actions that affect mTOR signaling and/or autophagy.

In conclusion, the present study demonstrates that everolimus may be used as a novel radiosensitizer in Ras-transformed cells, and that the mechanisms responsible for the everolimusmediated radiosensitization are partially dependent on the autophagy pathway.

\section{Acknowledgements}

The present study was supported by the Buddhist Dalin Tzu Chi General Hospital [DTCRD102 (2)-I-15].

\section{References}

1. Kolch W: Ras/Raf signalling and emerging pharmacotherapeutic targets. Expert Opin Pharmacother 3: 709-718, 2002.

2. Bos JL, Fearon ER, Hamilton SR, et al: Prevalence of ras gene mutations in human colorectal cancers. Nature 327: 293-297, 1987.

3. Kiaris H and Spandidos D: Mutations of ras genes in human tumors (Review). Int J Oncol 7: 413-421, 1995.

4. Bernhard EJ, McKenna WG, Hamilton AD, et al: Inhibiting Ras prenylation increases the radiosensitivity of human tumor cell lines with activating mutations of ras oncogenes. Cancer Res 58: 1754-1761, 1998.

5. Russell JS, Lang FF, Huet T, et al: Radiosensitization of human tumor cell lines induced by the adenovirus-mediated expression of an anti-Ras single-chain antibody fragment. Cancer Res 59: 5239-5244, 1999.

6. Gupta AK, Bakanauskas VJ, Cerniglia GJ, et al: The Ras radiation resistance pathway. Cancer Res 61: 4278-4282, 2001. 
7. Budde K, Lehner F, Sommerer C, et al: Conversion from cyclosporine to everolimus at 4.5 months posttransplant: 3 -year results from the randomized ZEUS study. Am J Transplant 12: 1528-1540, 2012.

8. Yunokawa M, Koizumi F, Kitamura Y, et al: Efficacy of everolimus, a novel mTOR inhibitor, against basal-like triple-negative breast cancer cells. Cancer Sci 103: 1665-1671, 2012.

9. Wolin EM: PI3K/Akt/mTOR pathway inhibitors in the therapy of pancreatic neuroendocrine tumors. Cancer Lett 335: 1-8, 2013.

10. Fu SL, Huang YJ, Liang FP, et al: Malignant transformation of an epithelial cell by v-Src via $t v-a$-mediated retroviral infection: a new cell model for studying carcinogenesis. Biochem Biophys Res Commun 338: 830-838, 2005.

11. Samid D, Miller AC, Rimoldi D, Gafner J and Clark EP: Increased radiation resistance in transformed and nontransformed cells with elevated ras proto-oncogene expression. Radiat Res 126: 244-250, 1991

12. Chen LT and Whang-Peng J: Current status of clinical studies for colorectal cancer in Taiwan. Clin Colorectal Cancer 4: 196-203, 2004.

13. Ito $\mathrm{H}$, Daido $\mathrm{S}$, Kanzawa $\mathrm{T}$, Kondo $\mathrm{S}$ and Kondo $\mathrm{Y}$ : Radiation-induced autophagy is associated with LC3 and its inhibition sensitizes malignant glioma cells. Int J Oncol 26 : 1401-1410, 2005

14. Okada $\mathrm{H}$ and Mak TW: Pathways of apoptotic and non-apoptotic death in tumour cells. Nat Rev Cancer 4: 592-603, 2004.
15. Cavazzoni A, Bonelli MA, Fumarola C, et al: Overcoming acquired resistance to letrozole by targeting the $\mathrm{PI} 3 \mathrm{~K} / \mathrm{AKT} / \mathrm{mTOR}$ pathway in breast cancer cell clones. Cancer Lett 323: 77-87, 2012.

16. Endo M, Yamamoto H, Setsu N, et al: Prognostic significance of $\mathrm{AKT} / \mathrm{mTOR}$ and MAPK pathways and antitumor effect of mTOR inhibitor in NF1-related and sporadic malignant peripheral nerve sheath tumors. Clin Cancer Res 19: 450-461, 2013.

17. Majumder PK, Febbo PG, Bikoff R, et al: mTOR inhibition reverses Akt-dependent prostate intraepithelial neoplasia through regulation of apoptotic and HIF-1-dependent pathways. Nat Med 10: 594-601, 2004.

18. Strimpakos AS, Karapanagiotou EM, Saif MW and Syrigos KN The role of mTOR in the management of solid tumors: an overview. Cancer Treat Rev 35: 148-159, 2009.

19. Wang L, Rhodes CJ and Lawrence JC Jr: Activation of mammalian target of rapamycin (mTOR) by insulin is associated with stimulation of 4EBP1 binding to dimeric mTOR complex 1. J Biol Chem 281: 24293-24303, 2006.

20. Balakumaran BS, Porrello A, Hsu DS, et al: MYC activity mitigates response to rapamycin in prostate cancer through eukaryotic initiation factor 4E-binding protein 1-mediated inhibition of autophagy. Cancer Res 69: 7803-7810, 2009.

21. Nyfeler B, Bergman P, Triantafellow E, et al: Relieving autophagy and 4EBP1 from rapamycin resistance. Mol Cell Biol 31: 2867-2876, 2011. 